Horizons philosophiques

\title{
Fondamentalisme et modernité
}

\section{Les trois monothéismes et les impasses de la raison}

\section{Georges Leroux}

Volume 13, numéro 1, automne 2002

Religion et pluralisme

URI : https://id.erudit.org/iderudit/801225ar

DOI : https://doi.org/10.7202/801225ar

Aller au sommaire du numéro

Éditeur(s)

Collège Édouard-Montpetit

ISSN

1181-9227 (imprimé)

1920-2954 (numérique)

Découvrir la revue

Citer cet article

Leroux, G. (2002). Fondamentalisme et modernité : les trois monothéismes et les impasses de la raison. Horizons philosophiques, 13(1), 71-89.

https://doi.org/10.7202/801225ar d'utilisation que vous pouvez consulter en ligne.

https://apropos.erudit.org/fr/usagers/politique-dutilisation/ 


\section{FONDAMENTALISME ET MODERNITÉ \\ LES TROIS MONOTHÉISMES ET LES IMPASSES DE LA RAISON}

Le scandale des monothéismes n'a pas cessé d'être un scandale politique. Moïse au Sinaï, Jésus au Temple, Mahomet à Médine n'ont jamais été les promoteurs d'une foi dégagée de l'histoire, ils ont au contraire élaboré un rapport de la foi à l'au-delà qui utilise pour se construire le levier même de l'histoire. À plusieurs égards, la notion d'une histoire sainte ou d'une histoire du salut, pensée par saint Augustin comme histoire unique des deux cités, constitue un héritage commun des religions du Livre. Cet héritage peut-il aujourd'hui être pensé sur l'horizon de la paix, ou demeure-t-il encore associé aux conflits qui ont marqué sa transmission? Les luttes fratricides des peuples du Livre, Juifs, Palestiniens, chrétiens sont irréconciliables avec l'origine commune de la doctrine du Dieu unique, et pourtant elles se perpétuent au nom même de la religion. Aux yeux de plusieurs, et en particulier aux yeux de penseurs séculiers, héritiers des Lumières, c'est le monothéisme lui-même qui engendre ces guerres: la volonté exacerbée de capter, pour se l'approprier, l'origine, serait pour chacune des trois traditions la raison de la violence historique.

Dans la résurgence actuelle du fondamentalisme religieux, un fait qui caractérise de manière égale les trois religions monothéistes, il faudrait voir l'aboutissement d'une violence originaire, faite d'un refus du partage de l'origine. Cette lecture n'est elle-même pensable que si les monothéismes sont figés dans la position crispée que recherche pour chacun le fondamentalisme, dont le principe est double : garantir l'autonomie des traditions, en bloquant tout ce qui pourrait concourir à leur unification, et figer l'interprétation des textes fondateurs pour neutraliser leur pouvoir de rédemption politique dans un effort de réconciliation. Ce ne sont donc pas les monothéismes en tant que tels qui sont la cause de la violence, mais leur captation par un fondamentalisme aujourd'hui en pleine expansion. Si le fondamentalisme est la vérité du religieux, son exposition essentielle, comme le prétendent tant de religieux aujourd'hui, il devient alors urgent de le penser et de le critiquer comme formulation de la violence et de l'engagement 
politique des religions. Retourner aux fondements, c'est en effet d'abord vouloir rompre avec ce qui est perçu comme un processus de dilution dans la modernité; c'est aussi vouloir mettre un frein au procès herméneutique qui travaille à relire les textes fondateurs pour rendre raison de leur commune origine et tenter de restituer à chaque tradition son autonomie et ses principes de lecture.

À plusieurs égards, le fondamentalisme religieux représente la position radicalement symétrique de celle qui fut mise de l'avant par la pensée des Lumières. Cette opposition peut être structurée comme une antinomie de l'autorité et de la raison, et c'est en ce sens, croit-on généralement, que le fondamentalisme affronte la modernité, c'est-à-dire comme une forme archaïque de l'autorité politique et religieuse. Cette opposition caractérise la pensée moderne et elle a contribué à la formation de la philosophie de la tolérance, chez des penseurs comme Pierre Bayle et John Locke : la tolérance peut tout tolérer sauf l'intolérant lui-même, rendant impossible par là même la fondation rationnelle du fondamentalisme. Seule la tolérance et le pluralisme, parce qu'ils sont fondés sur des arguments rationnels, sont acceptables; le recours à l'autorité, quel que soit son fondement, apparaît dans la modernité comme une position dépassée. Envisagé sur cet horizon, le fondamentalisme surgit comme désir de conserver les moyens de la violence, en conservant l'autorité d'une vérité du texte qui assure sa portée politique et son identité. Tout horizon de réconciliation lui paraît interdit.

Pourquoi la position philosophique des fondamentalismes n'est-elle pas tenable? Pourquoi personne ne s'adresse-t-il au fondamentalisme au nom de la nouvelle autorité de la raison? Selon Ernest Gellner, cette situation doit être imputée au nouveau contexte postmoderne de la rationalité. Ce contexte se caractérise par le relativisme, en passe de devenir la position qui se substitue au rationalisme dans la discussion avec le fondamentalisme. Toutes les positions religieuses sont en concurrence sur le marché de la croyance, quelles que soient leurs conséquences politiques et leur autorité, qui est d'abord une prétention à l'exclusivité de la vérité, n'est plus contestée par la raison, mais se trouve au contraire renforcée par le retrait de la raison devant l'émergence du pluralisme. Dans les pages qui suivent, je présenterai les thèses de Ernest Gellner, un anthropologue et philosophe britannique, sur la situation postmoderne et les arguments qu'il invoque pour un renforcement du rationalisme moderne. Je discuterai les aspects de ces thèses qui reposent sur 
une philosophie positiviste de la religion et j'essaierai de donner une formulation du relativisme qui soit susceptible d'intégrer une position pluraliste et tolérante.

En discutant avec Ernest Gellner, je voudrais d'abord rappeler deux éléments pour le situer dans un contexte plus large: d'une part, Gellner est un anthropologue dont l'œuvre savante a pris pour terrain l'organisation tribale des communautés musulmanes, en particulier au Pakistan. Son intérêt pour l'Islam n'est pas un intérêt superficiel, motivé comme on pourrait le croire, par la situation de tension croissante en Grande Bretagne entre les communautés provenant de l'immigration. Sa lecture du fondamentalisme est une lecture critique, produite à compter d'une vision pluraliste et séculière de la société. En second lieu, son œuvre philosophique en est venue à prendre plus d'importance que son œuvre scientifique: d'abord parce que, dans la foulée de l'épistémologie poppérienne, il a développé une critique radicale des sciences sociales; ensuite, parce que sa réflexion l'a conduit à proposer une théorie empirique du nationalisme qui a fait de lui un théoricien aussi important que son collègue Eric Hobsbawm. Le livre que je discute devait être à l'origine la première moitié d'un ensemble écrit en collaboration avec un penseur musulman, Akhbar Ahmed, mais les deux livres, suite aux difficultés de la discussion, ont été publiés séparément. J'en traiterai néanmoins comme d'un ensemble.

\section{Le concept du fondamentalisme}

Le concept de fondamentalisme auquel nous nous intéressons est d'abord un concept marqué par la question de la transcendance de la vérité. Plusieurs prédicats marquent cette transcendance et ils sont tous concomitants, aucun n'est dérivé d'une proposition originaire, compte tenu de l'importance de la notion de révélation. Par fondamentalisme, on désigne donc la position de ceux qui croient en l'existence éternelle d'une vérité universelle et qui croient qu'ils la détiennent en vertu d'une révélation. Cette définition, encore très imprécise, dans la mesure où elle ne comporte aucun déterminant historique ou politique autre que celui de la prophétie, s'applique adéquatement aussi bien à l'Islam qu'au judaïsme et au christianisme. Pour les trois traditions des religions du Livre, elle heurte cependant de front la notion de révélation qui s'est dégagée dans la modernité, où elle se trouve toujours assujettie au processus historique de 
l'interprétation. La notion d'un fondamentalisme est moins déterminante ou moins explicite que celle d'un intégrisme, qu'on pourrait définir comme suit : position de ceux qui dans l'exercice de la vie religieuse attachent une importance absolue au respect de l'intégrité de la doctrine et de la loi qui en découle. L'intégrisme peut ne pas s'accompagner d'une doctrine cohérente concernant le fondamentalisme, de la même manière qu'un fondamentalisme peut ne pas avoir de corollaire intégriste.

L'histoire de la philosophie politique et religieuse, aussi bien dans le monde chrétien que dans le monde musulman, a produit plusieurs tentatives pour tempérer le fondamentalisme et lui fournir des conditions d'interprétation susceptibles de respecter ses exigences radicales sur le plan de la vérité, tout en procurant une certaine détente sur le plan de ses conséquences politiques et morales. Cette détente entre la théologie politique héritée de saint Augustin d'une part, et la formulation de la Loi d'autre part s'est souvent opérée par le moyen d'un certain scepticisme. Les trois monothéismes ont connu plusieurs phases où leur philosophie a produit un idéal rationnel, assez fort pour entrer en tension avec les demandes de la foi: Maimonide, Alfarabi, saint Thomas d'Aquin, pour ne nommer que les plus importants, n'ont jamais jugé que la foi pouvait ou devait, en vertu de son caractère fondamental, contraindre la raison. Ils n'ont jamais jugé non plus que l'autorité de la foi, si elle contredisait la raison, devait diriger les décisions politiques. Quand cette tension s'est brisée à l'époque des Lumières, la théologie politique en a ressenti durement les effets: le fondamentalisme s'est trouvé mis en face d'un rationalisme dans lequel le scepticisme jouait un rôle non négligeable. La plupart des penseurs des Lumières, de Rousseau à Kant, étaient croyants, mais ils ne croyaient pas qu'ils avaient accès de manière immédiate à l'objet de leur croyance. Pour eux, comme pour tout ce qu'il est convenu d'appeler la modernité, la médiation de la raison devenait une tâche historique : la vérité existait, étemelle et universelle, mais il appartenait à l'humanité de la découvrir selon son rythme et son expérience. Le travail de l'histoire imposait ses lois et la prétention politique des fondamentalismes paraissait privée de toute légitimité.

Le dix-neuvième siècle a constitué le développement de cette nouvelle polarité, la religion et la métaphysique ne renonçant nullement à leurs prétentions fondamentales, mais acceptant qu'elles soient médiatisées dans l'histoire. Les critiques les plus radicales, 
celles de Ludwig Feuerbach par exemple, nous apparaissent aujourd'hui moins importantes que celles de Herder, dans la mesure où la considération de l'histoire introduite par l'anthropologie n'était assortie d'aucune perspective réductiviste ou éliminationniste.

Selon plusieurs théoriciens, et Ernest Gellner appartient à cette catégorie, la résurgence du fondamentalisme n'est cependant pas attribuable à ce qu'on pourrait appeler une arrogance des Lumières, ou à la domination de la raison. C'est la confiance même en la raison, c'est-à-dire, l'effritement du programme rationaliste qui a renforcé les perspectives du désenchantement mises en place par la sociologie de Max Weber, reprises aujourd'hui par Marcel Gauchet : le postmodernisme constitue précisément cette position de remplacement, qui vient poser devant le fondamentalisme aussi bien que devant le rationalisme, un relativisme généralisé, hérité de Nietzsche. Dans cette position, aucune vérité n'est disponible autrement que sur le mode de l'expérience singulière et aucune universalisation n'est pensable. Les communautés sont livrées à elles-mêmes, dans les aléas de leur expérience historique. Si donc on suit l'approche de Gellner, ce n'est pas tant le rationalisme des Lumières, et ce qui serait son programme réductiviste, qui serait à la racine de la résurgence des fondamentalismes, qui seraient alors à concevoir comme des réactions défensives et crispées, mais bien plutôt le déclin du rationalisme, qui libère un espace de concurrence, et en particulier un espace politique d'affrontement, que les fondamentalismes viennent occuper pour des raisons qui sous couvert de légitimité religieuse, poursuivent d'abord des finalités d'appropriation, d'identité et sont donc d'ores et déjà de nouvelles théologies politiques.

\section{Fondamentalisme, rationalisme, relativisme}

Ce rapide abrégé de l'histoire de la théologie politique nous amène au seuil d'une première proposition mise de l'avant par Ernest Gellner : dans cette évolution, qui est passée d'une polarité binaire héritée du Moyen Âge, dont le paradigme était celui de l'opposition de la raison et de la foi, à un schéma tripolaire, hérité du dix-neuvième siècle, un paradigme où la raison et la foi affrontent un tiers, qui est leur contradiction propre, le relativisme. Dans ce nouveau paradigme, aucune position n'est réductible à aucune autre. Le relativisme qui caractérise la post-modernité n'est pas une exagération du rationalisme, il n'en accomplit aucunement les virtualités intrinsèques : il s'agit d'autre chose, d'un programme qu'aucun penseur des 
Lumières n'aurait pensé possible. La confiance dans les pouvoirs de la raison, en particulier dans le domaine de la théologie politique, paraissait au dix-huitième siècle entièrement compatible avec une position de croyance et la plupart des philosophes qui ont préparé l'avènement des Lumières - on a cité John Locke et Pierre Bayle -, auraient été scandalisés qu'on puisse percevoir leur position comme posant les prémisses d'un athéisme ou même d'un relativisme, même si, et cela est surtout vrai de Bayle, ils ont soutenu qu'aucun argument rationnel ne pouvait être soutenu sous l'effet de la contrainte de la croyance. Leur effort a consisté à séparer deux sphères de la vie de l'esprit - la foi et la raison, l'Église et la société civile -, dans l'espoir d'apporter les bases de la paix politique. En cela, ils n'étaient que fidèles aux penseurs tolérants qui les avaient précédés, comme Maïmonide ou Marsile de Padoue et Nicolas de Cues.

Leur approche du fondamentalisme a d'abord consisté en la promotion d'un idéal de tolérance, qui en constituait justement la critique, et c'est cet idéal qui peut nous servir pour comprendre comment le rationalisme s'est opposé au fondamentalisme, tout en partageant avec lui un certain nombre de points majeurs, notamment la certitude de l'existence de la vérité. Dans cette approche de la religion, l'autorité du texte constitue le fondement de la doctrine et commande plusieurs corollaires : d'abord, l'interprétation du texte fondateur doit être littérale, de manière à bloquer toute interprétation morale ou spirituelle qui prendrait le risque d'engager le texte vers une ouverture à d'autres traditions, ou vers une réconciliation avec la raison. Un second corollaire concerne le lien intrinsèque, si important dans le judaïsme et dans l'Islam, entre le théologique et le juridique : le continuum de la doctrine et de la loi est dans tout fondamentalisme rigoureux, et ce trait montre ce qu'est le fondamentalisme chrétien en particulier, c'est-à-dire la résurgence de la loi dans une doctrine qui s'était posée à l'origine comme une ouverture de la loi.

Même s'ils repéraient beaucoup d'absurdités et d'incohérences dans la tradition chrétienne comme dans la tradition musulmane, les rationalistes n'y voyaient aucunement un argument contre les religions qui les incorporaient, seulement un défi pour la raison. Toutes ces traditions avaient produit en leur sein une école rationaliste, souvent dès leur période de première diffusion, comme on le voit par exemple à Alexandrie pour le christianisme, destinée à éclairer le contenu de la doctrine, et même dans les cas où ce 
rationalisme avait conduit à des positions extrêmes, la doctrine s'en était trouvée enrichie plutôt que déstabilisée.

La modernité a donc adopté à l'endroit des doctrines fondamentales une attitude à la fois critique et tolérante. Plusieurs philosophes se sont reconnus dans le projet latitudinaire de Locke, c'est-à-dire dans l'idée de restreindre le fondamental à un noyau minimal pour la raison, et de laisser à chaque croyance la responsabilité de maintenir son corpus doctrinal et d'élaborer sa loi. Mais ce projet, faut-il le rappeler, se développait sur le terrain même de la théologie politique, puisque tout ce qu'il retirait des prétentions de la doctrine fondamentale, il le confiait à l'Etat. Plus la promotion de l'idéal de tolérance s'est étendue, plus il est devenu difficile à la théologie politique d'élaborer ses bases universelles. II ne faut jamais oublier que cette discussion s'est faite de manière très concrète, notamment en Angleterre et aux Pays-Bas; c'est lentement que le magistère chrétien a concédé qu'en Europe le rationalisme avait transformé le fondement de la vie politique et qu'il tempérait désormais de manière essentielle la doctrine chrétienne. Dit autrement, c'est le fruit d'une longue histoire que celle de l'abandon progressif dans le christianisme européen des positions fondamentalistes, essentiellement augustiniennes, qui avaient donné leur fondation à la théocratie et aux monarchies de droit divin.

Par contraste avec la tradition chrétienne, on ne peut pas parler d'un modernisme rationaliste pour l'Islam. Plus la théologie musulmane s'est approfondie, plus elle s'est éloignée de l'idéal rationaliste de l'litihad qui avait caractérisé son âge d'or médiéval. La domination de l'interprétation du texte coranique dans les hadith, la formulation de la charia par l'autorité, ont écarté en quelque sorte l'idéal d'un consensus communautaire rationnel et diminué en proportion la place de la philosophie. Ces deux traditions ont vécu dans des espaces entièrement séparés, en dépit d'échanges politiques et commerciaux importants, rompant avec une pratique très riche au Moyen Âge et à la Renaissance. On pense à Pierre Le Vénérable, à Pierre Abélard et surtout à Raymond Lulle, tous admirateurs de la culture musulmane et désireux de communiquer avec elle sur le plan de la philosophie. Au dix-huitième siècle, I'Islam est devenu l'autre absolu, incommensurable, l'envers même de la modernité. L'article Mahomet de l'Encyclopédie en constitue le meilleur exemple. 
Gellner attache néanmoins une certaine importance aux conséquences des Lumières dans l'évolution du fondamentalisme. En Europe, celui-ci n'a pas été entièrement annulé. Son programme était sans doute impraticable et il a donné lieu à plusieurs édulcorations qui sont surtout le fait du dix-neuvième siècle : si la raison est véritablement distincte de la foi, elle ne sera donc pas troublée par les absurdités ou les incohérences logiques des religions. Cette idée, si bien représentée par le credo quia absurdum de Tertullien, est répercutée par exemple chez Kierkegaard : la foi est un engagement, une dévotion, et elle ne doit pas attendre que la raison lui dicte la conduite à tenir. Dit autrement, on peut contourner l'affrontement moderne en le radicalisant, et dès lors on peut retrouver un certain fondamentalisme. La position de Kierkegaard est fondée sur un paradoxe qui sera néanmoins inacceptable pour un fondamentaliste, en raison du peu de cas qu'elle fait du texte dans son rapport à l'autorité.

Cette position nous met en face d'un trait majeur de tout fondamentalisme: il répudie absolument tout modernisme, c'est-à-dire tout accommodement interprétatif tendant à réconcilier le texte doctrinal avec les convictions de l'époque. Par exemple avec la science. Les discussions sur la pensée de Darwin aux États-Unis sont de bons exemples de cet affrontement : là où Kierkegaard ou des croyants modernes pensent que le texte biblique ne s'oppose pas à la proposition scientifique, puisqu'il en est en quelque sorte l'expression symbolique, le fondamentaliste refuse toute espèce de médiation symbolique, toute herméneutique.

Le processus contemporain de la sécularisation a donc structuré l'opposition du fondamentalisme et du rationalisme, dans la mesure où le travail de la raison a beaucoup emprunté les chemins de l'herméneutique et alimenté tous les compromis modernistes que le dix-neuvième siècle avait mis en chantier. Pendant plus d'un siècle, d'Auguste Comte à maintenant pour faire court, il ne semblait ni possible, ni souhaitable d'interpeller la doctrine religieuse comme on le faisait au dix-huitième siècle, c'est-à-dire en lui demandant des comptes au nom de la raison. Le monde de la religion était un autre monde, symboliquement distinct. L'attitude kierkegaardienne rendait pensable le paradoxe moderne de la foi et justifiait en même temps toutes les stratégies modernistes de l'herméneutique. Aucun penseur 
contemporain ne semble véritablement exempt de ces attitudes de compromis, et leur légitimité ne saurait être mise en question. Leur exemple sert seulement de repoussoir pour comprendre le refus du compromis qui constitue le cœur du fondamentalisme.

Or il se trouve que le fondamentalisme, aussi bien en Europe, en Amérique que dans les pays musulmans, apparaît essentiellement lié au refus de la sécularisation. Cette proposition ne va pas de soi. Elle met en cause en effet l'ensemble des conséquences du rationalisme des Lumières, et plus particulièrement l'idéal de tolérance lié par Locke et Bayle à la séparation des sphères de la religion et du politique. La résurgence d'un idéal de théologie politique, profondément hégémonique de nature, n'a certainement pas la même force dans le judaïsme, dans le christianisme et en Islam.

\section{L'exemple de I'Islam}

La thèse principale de Gellner est que I'Islam constitue une exception au processus universel de la sécularisation. Cette exception est-elle inexplicable? Le processus de la sécularisation, comme accomplissement de l'idéal des Lumières, est-il un processus immanent de l'histoire universelle? Pour discuter correctement cette position, il n'est peut-être pas nécessaire d'accepter les prémisses positivistes de la thèse. Gellner croit certainement juste et nécessaire que les formes archaïques de l'autorité, fondées sur le rituel et la révélation, cèdent la place à la seule autorité de la raison. Sa position ne concède aucun rôle, aucune fonction positive aux religions dans la société contemporaine et pour lui le processus de sécularisation consiste en une élimination progressive du religieux. Dans cette perspective, le fondamentalisme est donc une réaction naturelle à cette érosion de l'autorité.

II semble important de préciser ici que la théorie de la sécularisation, bien représentée dans l'École de Francfort, est par ailleurs percutée de front dans la philosophie contemporaine actuelle par un retour considérable de la théologie politique. Je ne pense pas seulement à Léo Strauss, lecteur de Platon, de Maïmonide et de Spinoza, mais à une nouvelle génération de philosophes inquiets de l'impossibilité d'une fondation non-théologique du politique. Je n'accepterai donc pas d'emblée l'idée que le processus général de la sécularisation soit considéré comme la raison ultime du regain fondamentaliste. II faut une analyse plus fine. 
Cette approche ne nous empêche pas de discuter l'exemple de I'Islam pour ce qu'il est: une illustration d'une force extraordinaire du fondamentalisme dans sa composante de théologie politique. II ne sera pas nécessaire de passer en revue ici tous les traits de l'Islam qui l'identifient comme une position fondamentaliste : le point central est l'origine divine de la Loi, qui la rend immuable et qui fonde l'inséparabilité de la doctrine religieuse et du droit. C'est cette proposition qui se trouve à la base de la théologie politique du Coran. Les traditions qui séparent les musulmans (sunnites, chiites, kharejites) se fondent sur l'importance relative de l'autorité du Coran, dans son rapport au consensus de la communauté et à l'autorité sacrée de ses chefs. Les chiites révèrent l'autorité sacrée, et attendent le retour de l'imam caché; les sunnites sont plus légalistes.

En quoi consiste précisément le fondamentalisme musulman? Parce que l'Islam, victorieux politiquement dès le point de départ, a été très rapidement une religion et un État, la question du fondamentalisme a toujours été une question de théologie politique. Par comparaison, on peut penser à une théologie chrétienne qui serait éternellement augustinienne. Dans ce monde augustinien, Gellner distingue comme la plupart des experts un haut Islam, urbanisé et scolarisé et un Islam populaire (Low Islam, folk-Islam), plus campagnard et moins scolarisé. Cette catégorisation tend à séparer un Islam rationnel et discipliné, voué à l'étude de la loi, et caractérisé par le puritanisme et le scripturalisme, d'un Islam émotionnel, adonné à la magie et au culte des saints, soucieux de la médiation avec Dieu.

Le passage du fondamentalisme à l'intégrisme politique radical pose des questions redoutables, notamment parce que le recours à la violence politique y devient légitime. On peut penser que c'est cet Islam fondamentaliste qui est à la base des confréries comme les Frères musulmans et sans doute comme le Front islamique du salut algérien. Mais les transformations sont complexes et méritent des analyses fouillées. Dans l'analyse anthropologique de Gellner, les leaders savants utilisent souvent l'Islam populaire des fraternités pour venir corriger et nettoyer la corruption urbaine. Le cas actuel de l'Algérie n'est qu'un des exemples de cette dialectique du monde urbain et du monde rural qui structure le fondamentalisme; selon Gellner, on peut la généraliser dans l'histoire de l'Islam, ce qui permet de dégager une structure du rapport du fondamentalisme aux mouvements et aux cycles de la renaissance, dont témoignent tous les 
mouvements de Islamic Revival, comme El Qaida. Quand l'ardeur de la réforme s'apaise, I'Islam rural retourne à son mode de vie traditionnel et social. Mais la situation contemporaine récente semble vouloir contredire ce cycle socio-politique: l'Islam populaire a perdu sa légitimité en faveur de l'Islam scolarisé des villes. Le fondamentalisme d'une religion sans médiation a triomphé d'un projet de médiation rituel.

Cela se voit dans plusieurs facteurs qui influencent le développement du fondamentalisme: le déclin des sanctuaires et des cultes, le recours à la loi des Lettrés, le rôle accru des universités et la croissance du nationalisme politique comme nationalisme musulman. Dans cet Islam urbanisé, le rôle de la Loi est central et il alimente une conception puritaine et égalitaire des musulmans : chacun est appelé à se définir en face du texte et à se conformer à lui, sans passer par les médiations communautaires du sanctuaire ou de la tribu. Pour le comprendre, ce n'est pas d'abord l'Iran chiite qui peut nous aider, car on y trouve surtout un Islam des martyrs et de l'effervescence politique assez éloigné du fondamentalisme légaliste. C'est l'Islam maghrébin qui illustre le mieux la révolution culturelle de I'Islam contemporain. Mais dans les deux cas, le fondamentalisme a soutenu un mouvement intérieur de réforme, parfois appelé révolution.

Notre tâche est d'interpréter le rapport entre le fondamentalisme et la violence politique: si l'Islam constitue une exception véritable au processus de la sécularisation, est-ce en raison d'une résistance doctrinale aux valeurs du rationalisme, ou est-ce en raison d'une situation économique et politique qui utilise le fondamentalisme pour se légitimer? Dans l'approche de Gellner, le fondamentalisme est instrumental; il sert à cautionner un mouvement de réforme dans des situations nationales faibles sur le plan économique. La crainte de la domination occidentale a provoqué non pas le retour vers un Islam mystique ou populaire, mais la revitalisation de I'Islam lettré. La révolution des saints a laissé la place à une véritable mutation culturelle qu'on peut résumer ainsi: il est devenu possible de s'identifier, pour tous, à l'Islam puritain des lettrés : ce qui n'était auparavant que le fait d'une minorité, sous l'effet d'une profonde impulsion morale de réforme, est étendu à tous.

Comment caractériser ce fondamentalisme contemporain? C'est une idéologie puritaine, auto-correctrice, soucieuse de guérison et ce sont ces caractéristiques qui favorisent son évolution vers l'intégrisme radical et la violence politique. Le fait de recourir à un modèle 
intrinsèque, profondément ancré dans l'histoire de l'Islam, présente de grands avantages. Le plus important est de justifier une certaine rigueur économique à compter de l'histoire nationale, c'est-à-dire de l'histoire sainte de l'État national. Sa conviction principale est qu'il est possible de connaître une vérité universelle transhistorique, notamment concernant la morale et le sens de la vie, le fondement du pouvoir et l'origine divine de la Loi. Même si le fondamentalisme n'a pas intégré les conquêtes du rationalisme des Lumières, on peut donc soutenir qu'il n'est pas radicalement étranger aux convictions kantiennes. La résistance à la sécularisation n'est pas tant une résistance à la raison qu'un refus du pluralisme et de l'occidentalisation.

Cette situation explique que son véritable opposé soit le relativisme, c'est-à-dire la doctrine selon laquelle aucune connaissance de cette nature n'est accessible sur un mode autre que subjectif. Dans son analyse, Gellner se livre à une charge assez facile contre le post-modernisme en anthropologie sociale, qu'il identifie à une fascination paralysante pour l'Autre, qui finit par faire renoncer à la recherche de toute objectivité et de toute universalité. Je laisse de côté cet aspect de son propos, pour m'en tenir à son argument principal: le regain fondamentaliste est une réaction non pas au rationalisme occidental, mais à l'effondrement de ce rationalisme.

II est en effet question de revenir à l'affrontement de la rationalité avec non pas le dépassement théologico-politique auquel elle fut toujours liée, mais avec le relativisme qui associe essentiellement le savoir et la morale à l'existence même de la culture singulière. Plusieurs discussions philosophiques actuelles mériteraient d'être citées ici, mais deux problèmes pourront être introduits pour en donner une idée: premièrement, si la morale (la Loi au sens musulman) est dépendante de la culture, elle est alors entièrement contingente et elle doit renoncer à sa fondation universelle; deuxièmement, existe-t-il des connaissances qui, comme les doctrines religieuses, sont au-delà des savoirs moraux contingents?

Un exemple qui permet de progresser est celui de l'égalité des hommes et des femmes : je le mentionne sans le discuter. Dans une perspective de tolérance, nous acceptons le voile, mais nous ne tolérerions pas l'affirmation déclarée de l'infériorité de la femme et de sa soumission. Dans la perspective d'une réelle tolérance moderne, nous devrions cependant le tolérer, puisque cette proposition morale fait partie du corps des connaissances contingentes. Mais nous ne le 
tolérons pas, parce que cette proposition nous semble heurter la raison. Or, dans le fondamentalisme musulman, cette proposition échappe au contrôle de la raison, et il faut reconnaître que cette position théologique est possible. L'Église catholique elle-même maintient cette position dans son interdiction de l'ordination des femmes. La plupart des philosophes intéressés par ces questions cherchent à déterminer si une proposition morale de ce genre peut être considérée comme un savoir, ou si elle appartient pour reprendre l'expression de John Rawls au fait du pluralisme. On en dirait autant de la dignité de la vie humaine dans la discussion de l'avortement. Ou encore de la légitimité de la démocratie. Toutes ces propositions sont percutées de front par le fondamentalisme, qui n'y reconnaît pas un savoir, mais une position morale non démontrée par le rationalisme. Si la raison renonce à les soutenir comme des propositions démontrables, et accepte de les proposer comme des propositions relatives et contingentes, elle crée en même temps un vacuum dans lequel le fondamentalisme trouve l'espace de ses propres certitudes. Telle est la dynamique, à la fois nouvelle et mal analysée, du relativisme.

Si nous suivons Gellner, nous voyons bien cependant qu'un relativisme moral et politique n'est pas le corollaire nécessaire du relativisme scientifique : il existe un savoir transculturel, ne serait-ce que celui des mathématiques et des lois de la logique, il y a donc un universel minimal. Au fur et à mesure que cet universel s'est développé, à compter du rationalisme grec et européen, la religion s'est détachée du corps social, le monde s'est engagé dans un processus de sécularisation et le transcendant s'est isolé. Le rationalisme est en ce sens le successeur du monothéisme judéo-chrétien. Cette proposition laisse de côté les aspects problématiques de la métaphysique (l'immortalité de l'âme, le fondement de la morale), mais depuis Kant le destin de la science s'est érigé comme le seul véritable interlocuteur du fondamentalisme.

La situation moderne se crispe cependant précisément sur ce destin universel du rationalisme: dans la mesure où elle transcende les cultures, la science leur est totalement indifférente. Le consensus des chercheurs est le seul élément nécessaire à son progrès. On voit donc comment sur deux fronts différents ce rationalisme scientifique vient restructurer la discussion. D'une première façon, devant l'interlocuteur religieux, soucieux de la communauté, le rationalisme paraît indifférent aux normes; il libère donc la prétention fondamentaliste à formuler les normes. Encore sur ce point, le 
fondamentalisme n'est pas en contradiction avec le rationalisme, au contraire il peut s'en alimenter, dans la mesure où il occupe toutes les places laissées vides par la science sur les questions fondamentales du lien social et du destin religieux. La première limite du rationalisme est donc en un sens son abandon de la métaphysique et son renoncement au savoir moral.

Mais, et c'est le second enjeu, cet abandon et ce renoncement sont désormais théorisés dans l'édifice même de la rationalité, bien sûr à des degrés divers: le relativisme qui caractérise la post modernité vient donc renforcer l'urgence et la légitimité du projet fondamentaliste. Non seulement la raison accepte-t-elle de s'identifier au destin technologique de la science, en s'y limitant, mais elle renonce au projet des Lumières en acceptant le pluralisme, rendant ainsi nécessaire la relativité du savoir moral. C'est cette position ultime qui, bien plus que le rationalisme ou toute opposition de la foi et de la science, vient ouvrir l'espace du fondamentalisme, d'abord soucieux de restaurer l'unité des certitudes perdues.

Dans la perspective de l'Islam, toutes les sociétés riches sur le plan technologique ont renoncé à la maîtrise de leur évolution morale; leur prétention à la domination est une illusion, autant pour ellesmêmes que pour les sociétés qu'elles prétendent asservir. Cette leçon est proposée non seulement par le fondamentalisme, mais par tous les penseurs relativistes qui dans la culture occidentale refusent de justifier le rationalisme scientifique précisément parce qu'il produit une indifférence au savoir moral, et dans certains cas extrêmes, parce qu'il est lui-même relatif. Ce relativisme vient donc, étrangement il faut le reconnaître, soutenir la prétention fondamentaliste: si la raison échoue sur l'essentiel, pourquoi ne pas reconnaître le privilège unique de la révélation et lui accorder le statut d'un fondement pour le savoir moral aussi bien que pour l'organisation politique?

Il est temps de récapituler notre discussion : comment les fondamentalismes dont nous observons la résurgence trouvent-ils leur légitimité? Notons en premier lieu que la sécularisation est la conséquence du développement du rationalisme techno-scientifique; elle rend possible l'autonomie de la sphère politique et de la sphère religieuse, tant sur le plan de la formulation des normes que sur celui des institutions. Notons dans la foulée que le rationalisme devient indifférent aux questions morales ou religieuses, de même qu'à l'organisation politique; il n'a rencontré aucun succès dans le domaine des sciences sociales et les efforts de naturaliser les normes ont 
trouvé rapidement leurs limites, laissant aux religions et aux systèmes moraux qui leur sont associés la tâche de les proposer. Par ailleurs, le rationalisme a été contesté de l'intérieur par le post-modernisme relativiste, sur deux plans: d'abord, de manière faible sur le plan de la connaissance, mais surtout de manière forte sur le plan des systèmes de culture et de croyance. Le fondamentalisme n'est pas tant, dans cette perspective, la résistance au monde de la science, que le refus de toute relativisation du savoir moral, de nature théologico-politique. S'il accepte que l'univers cognitif de la science soit universel, dans la mesure où il n'interfère pas avec le savoir moral, il refuse par ailleurs toute relativisation du texte et de l'autorité de l'écriture qui déstabiliserait les normes et paralyserait l'autorité politique qui en tire sa justification. Un bon exemple de cela est le fait que le repli sur le puritanisme et le Haut Islam du scripturalisme est entièrement compatible avec le rationalisme, mais absolument incompatible avec le relativisme.

Cette discussion peut s'élaborer si on y inclut la réflexion de penseurs comme A. Ahmed qui en conteste les prémisses. Là où Gellner déplore l'abandon du programme des Lumières devant l'extension du pluralisme et veut faire la promotion d'une véritable révolution rationaliste susceptible de triompher du fondamentalisme en énonçant des valeurs universelles, Ahmed pense au contraire que le fondamentalisme appartient au monde de la post-modernité, qu'il en constitue un des traits les plus féconds et qu'il s'accorde avec ses prémisses relativistes.

Les chemins se séparent donc ici entièrement du rationalisme scientifique de Gellner et de ce qu'on pourrait appeler son positivisme hostile à toute expérience religieuse. Le postmodernisme réside en effet dans l'affirmation d'un véritable pluralisme: sceptique à l'endroit des orthodoxies traditionnelles, il est aussi critique à l'endroit de l'optimisme des Lumières. Comment concilier cela avec le fondamentalisme musulman? La réponse de Ahmed est aussi claire que simple : "Where nothing is sacred, every belief becomes revisable. Thus fundamentalism is the attempt to resolve how to live in a world of radical doubt...ethno religious revivalism is both cause and effect of postmodernism.»

Mais quand nous lisons ses analyses, nous sommes d'abord saisis par le fait que, pour lui, post-modernisme signifie tolérance et optimisme, et qu'il se sépare du post-modernisme occidental, s'il doit connoter un cynisme qui refuse la raison. La vraie question, qui est 
celle de l'origine de la Loi et des normes, demeure donc chez ce penseur suspendue dans le vide.

Ces deux points mériteraient chacun un traitement séparé, puisque chacun réfère à une signification particulière du fondamentalisme. Sur ces deux plans cependant, les principes sont les mêmes: primauté de la révélation coranique, priorité de la morale sur tous les aspects de la vie. Pour entrer dans la discussion contemporaine de ce fondamentalisme, il faut lire les principaux théoriciens: les traditionalistes, les modernistes et les radicaux. Pour les traditionalistes (Hossein Nasr, Al Faruqi, Fazlu Rahman), l'expérience de la foi est centrale et la primauté du texte coranique indiscutable. Malgré cela, le dialogue interconfessionnel est possible et l'Islam traditionnel conserve un message de tolérance et un intérêt pour la spiritualité et la mystique. Par comparaison, les penseurs radicaux (Parveez Manzoor), comme toute la cohorte de ceux qui favorisent le passage vers l'intégrisme, se concentrent sur l'importance de l'action politique. Pour eux, le cœur de l'Islam est la théologie politique et leur message n'est pas tolérant, il élabore surtout une critique de l'occidentalisation, fondée en grande partie sur une idéalisation d'une société perdue. Les penseurs modernistes (Tariq Ali, Rushdie) sont moins présents dans la discussion, mais leur rôle est essentiel. Adeptes de la sécularisation et du compromis, ils ne sont pas nécessairement relativistes. Interprètes démythologisants du Coran, ils s'engagent dans le renouvellement de l'herméneutique et comme beaucoup de penseurs chrétiens, ils croient que le noyau central de l'Islam est sa doctrine morale.

Dans cet ensemble, on voit que la dernière catégorie est d'emblée paradoxale : que peut vouloir dire un musulman séculier, un fondamentalisme critique? C'est pourtant la position de Rushdie. Le monde musulman intellectuel est extrêmement tendu et crispé devant l'enjeu fondamentaliste, devant l'urgence de réfléchir ce retour aux valeurs originelles de l'Islam pour éviter les dérives politiques radicales. Même les traditionalistes pensent que les modernistes et les radicaux devraient se joindre à eux et retourner aux sources, de manière à dépasser les stéréotypes négatifs qui les accablent. Ces sources sont l'exégèse du Coran, mais aussi l'litihad, c'est à dire la recherche du consensus rationnel.

Dans l'espace de la discussion post-moderne, on entend donc de plus en plus une demande de réintroduire la rationalité. L'essai devenu célèbre de Adelrazziq sur la séparation des pouvoirs, 
unanimement critiqué et condamné lors de sa parution, pourrait devenir le texte fondateur du nouvel Islam post-moderne. Un équivalent de Locke ou de Bayle pour notre culture philosophique occidentale.

Mais cette discussion ne pourra progresser que si les enjeux de la situation que j'ai tentée de résumer sont clarifiés : 1- le fondamentalisme, dans toutes ses variantes, profite du vacuum produit par le post-modernisme occidental; il s'en accommode, mais peut parfois se retourner contre lui, comme dans l'affaire Rushdie. Ceci l'expose au paradoxe de sa propre intolérance : intolérant envers Rushdie, il demande néanmoins qu'on le tolère. Un des acquis de la rationalité moderne est de ne pas accepter cette position; $2-$ le fondamentalisme s'est éloigné de ses sympathies rationalistes; ce qu'il partage avec le rationalisme (la croyance en une vérité qui ne dépend pas de la culture) devrait pourtant l'en rapprocher; 3 - mais le rationalisme partage avec le post-modernisme la croyance dans l'idée qu'il n'existe pas de réponse absolue, donnée de manière transcendante; dans la perspective du rationalisme, tout savoir est faillible, révisable.

Tout l'enjeu du dialogue entre ces positions philosophiques peut être résumé comme l'impasse contemporaine de la raison : comment la raison peut-elle en effet croire qu'il existe un savoir qui ne soit pas contingent, et en même temps nier l'origine divine qui est centrale pour le fondamentalisme? La réponse à cette question ne peut être que l'affirmation de la supériorité de la science occidentale sur le plan formel ou procédural; que cette supériorité puisse être étendue à la philosophie, dans sa volonté de rationaliser l'expérience morale et politique est bien entendu entièrement discutable, mais cette volonté appartient à notre culture. Mais ici nous butons sur l'écueil monumental de la croyance et de la métaphysique: s'il n'y a aucune vérité substantielle ou a priori, il n'y a donc rien de sacré ou d'absolu dans notre monde. Un rationaliste comme Gellner l'affirmera nettement, et il se situera dans l'héritage des Lumières, même si, comme je l'ai dit au début, rien dans la pensée des Lumières n'excluait la proposition transcendante ou la métaphysique.

Pour intégrer le fondamentalisme dans notre vision du monde, pour lui faire une place, il faut donc accepter de limiter l'extension du rationalisme scientifique et ses prétentions; il faut aussi considérer le processus de la sécularisation comme contingent, ce que refuse Gellner. Cette position conduit cependant à une intolérance qui aurait été inacceptable à l'époque des Lumières et qui doit l'être encore 
aujourd'hui. Certes, l'idéal de tolérance est un idéal conquis rationnellement, mais dans la mesure où lui seul peut assurer la cohabitation du fondamentalisme et du rationalisme, dans un monde post-moderne, il est le seul à pouvoir être encore proposé aujourd'hui. Si on nie la possibilité même de la révélation, notamment pour affronter le fondamentalisme, on se place immédiatement dans une position intolérante; en retour, tous ceux qui adoptent la position de la révélation, doivent aussi accepter que chacune de leurs affirmations soit librement critiquée par l'exercice des procédures de la raison. Non pour s'exposer au cynisme et à la destruction, mais pour rendre possible une liberté qui est le fondement même de la tolérance. La raison elle-même ne doit-elle pas accepter d'être interpellée à la fois par la pluralité des significations dans les cultures et par la certitude de ceux qui sont attachés à des révélations?

Dans l'évolution actuelle de l'Europe et de l'Amérique, on peut penser que la vision du monde musulmane (mais aussi celle du fondamentalisme chrétien ou juif aux États-Unis) va produire une crispation du rationalisme que nous ne pouvions imaginer : non seulement l'universalité de la sécularisation semble désormais indémontrable, mais toutes les interprétations réductrices des mouvements de réforme (par exemple, les interprétations qui cherchent à les expliquer par des frustrations économiques) se montreront impuissantes devant les requêtes morales des individus et des communautés. Le fait du pluralisme des visions du monde, qui compose les trois grands modèles exposés par Gellner, est devenu une composante irréductible de l'existence contemporaine : chacun de ces modèles a des avantages évidents, mais aussi des faiblesses. Chacun se trouve en directe interlocution avec les autres, notamment par l'extension mondiale de la communication et il constitue, pour les traditions monothéistes, engagées dans une évolution politique complexe, le nouveau paradigme de leur interprétation.

\section{Georges Leroux \\ Département de philosophie, UQAM}




\section{Références bibliographiques}

AHMED, Akbar S \& HASTINGS, Donnan. Islam, Globalization and Postmodernity, London, Routledge, 1994.

ABDERRAZIQ, Ali. L'Islam et les fondements du pouvoir. Paris, La Découverte, “Textes à l'appui», 1994.

AHMED, Akbar S. Discovering Islam. Making Sense of Muslim Society and History. London, Routledge, 1988.

AHMED, Akbar S. Postmodernism and Islam. Predicament and Promise. London, Routledge, 1992.

ARKOUN, Mohammed, Pour une critique de la raison islamique. Paris, Maisonneuve et Larose, 1984.

DANIEL, Norman. Islam et Occident. Paris, Editions du Cerf, «Patrimoines», 1993.

FISCHER, Michael M. J \& Abedi, Mehdi.Debating Muslims : Cultural Dialogues in Postmodernity and Tradition, Madison,

University of Wisconsin Press, 1990.

GELLNER, Ernest. Muslim Society. Cambridge, Cambridge University Press, 1982.

GELLNER, Ernest. Postmodernism, Reason and Religion. London, Routledge, 1992.

NASR, Seyyed Hossein. Knowledge and the Sacred. Edinburgh, University Press, "The Gifford Lectures",1981,

RAHMAN, Fazlur. Islam and Modernity:Transformation of an intellectual Tradition. Chicago, Chicago University Press, 1984. 